April 1999

KEK-TH-622

hep-th/9904122

\title{
Cohomological Field Theory Approach to Matrix Strings
}

\author{
Fuminiko Sugino也 \\ Institute of Particle and Nuclear Studies, \\ High Energy Accelerator Research Organization (KEK), \\ Tsukuba, Ibaraki 305-0801, Japan
}

\begin{abstract}
In this paper we consider IIA and IIB matrix string theories which are defined by twodimensional and three-dimensional super Yang-Mills theory with the maximal supersymmetry, respectively. We exactly compute the partition function of both of the theories by mapping to a cohomological field theory. Our result for the IIA matrix string theory coincides with the result obtained in the infra-red limit by Kostov and Vanhove, and thus gives a proof of the exact quasi classics conjectured by them. Further, our result for the IIB matrix string theory coincides with the exact result of IKKT model by Moore, Nekrasov and Shatashvili. It may be an evidence of the equivalence between the two distinct IIB matrix models arising from different roots.
\end{abstract}

\footnotetext{
${ }^{1}$ E-mail address: sugino@post.kek.jp
} 


\section{Introduction}

A recent great development of nonperturbative aspects of superstring theory has begun with following two events. One is a proposal of M-theory [1], which is a hypothetical theory describing strongly coupled region of type IIA superstring theory and reduces to elevendimensional supergravity in the low energy limit. The relation among parameters of string theory (the string length $l_{s}$ and the string coupling $g_{s}$ ) and of eleven-dimensional supergravity (the Planck length $l_{11}$ and the radius of compactified the 11-th direction $R_{11}$ ) is given by

$$
R_{11}=g_{s} l_{s}=g_{s}^{2 / 3} l_{11}
$$

M-theory gives a systematic view point for string theory — in considering the M-theory, all known perturbative string theories are unified via various connections from duality. The other is a discovery of D-branes [2], which are solitonic solutions of string theory and can be constructed by using conformal field theory in a well-defined manner. Thus they are powerful tools for investigating nonperturbative phenomena in string theory. Further, it was followed by a new interpretation of dimensional reductions of ten-dimensional supersymmetric YangMills (SYM) theory as low energy effective theories for dynamics of the D-branes [3].

Those movements culminated in the conjecture by Banks, Fischler, Shenker and Susskind

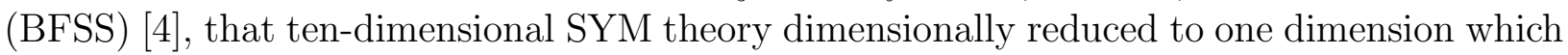
describes the low energy dynamics of D0-branes so far, in the infinite momentum frame, gives a constructive definition of the M-theory. This one-dimensional SYM quantum mechanical model is called M(atrix) theory. Moreover, by considering toroidal compactification on a circle and on a two-torus in the manner of Taylor [5], it leads to a proposal for a nonperturbative definition of type IIA superstring theory [6, 7] and that of type IIB superstring theory [7, 8], respectively. In this paper, we call them IIA and IIB matrix string theories. The IIA matrix string theory is given by ten-dimensional SYM theory dimensionally reduced to twodimensions, and the string coupling constant corresponds to the inverse of the SYM coupling. Also, the IIB matrix string theory is a dimensional reduction of the ten-dimensional SYM theory to three dimensions. The string coupling is given by a ratio of lengths of two spatial dimensions of the three-dimensional SYM theory. In addition, with respect to another nonperturbative definition of type IIB superstrings [9], which is in form of the ten-dimensional SYM theory reduced to a point (zero dimension), proposed by Ishibashi, Kawai, Kitazawa and Tsuchiya, it is referred to IKKT model.

Those matrix string theories successfully reproduce some of known results obtained from an analysis of BPS saturated states consisting of fundamental strings, D-branes and their bound states [10]. This fact is considered as one of evidences that the matrix string theories truely are constructive definitions of string theories. However, with respect to nonperturbative dynamics of the string theories, in order to investigate it we cannot help treating nonperturbative dynamics of the SYM theory sides. Though understanding of this area is now in development [11, 12], unfortunately at present it seems to be not powerful enough for handling the problem.

In this paper, we consider the partition function of the matrix string theories - it is one of 
the most fundamental quantities reflecting dynamical property of vacua of the theories. The partition function of the IIA matrix string theory was computed in strongly coupled limit of the SYM theory by Kostov and Vanhove [13]. For the partition function of the IKKT model, the exact result was obtained by interpreting the theory as a cohomological field theory by Moore, Nekrasov and Shatashvili 14. Here, we exactly calculate the partition function of both of IIA and IIB matrix string theories by mapping the theories to cohomological field theories. In the IIA case our result coincides with the result obtained in the strongly coupled limit, which thus gives a proof of exact quasi classics discussed in [13]. Also in the IIB case, our result agrees with the exact calculation for the IKKT model, which seems to suggest the equivalence between those two different nonperturbative formulations of IIB string theory.

The paper is organized as follows. In the next section, we introduce the IIA and IIB matrix string theories via toroidal compactification of the M(atrix) theory. We briefly explain how string interactions emerge from the SYM theory and show how spinors with correct chirality appear. In section 3, as a preparation for computing the partition function of the matrix strings, we evaluate the partition function of four-dimensional $\mathcal{N}=4 S U(N) \mathrm{SYM}$ theory defined on a four-torus by mapping the theory to a cohomological field theory via a twisting procedure. Then in section 4, we exactly compute the partition function of the IIA matrix string theory, and see that our result coincides with the result in the infra-red limit by Kostov and Vanhove. In section 5, for the partition function of the IIB matrix string theory we perform the calculation in the ten-dimensional IIB limit, and find the identical result with the partition function of the IKKT model by Moore, Nekrasov and Shatashvili. Finally, section 6 is devoted to conclusions. In Appendix A, we clarify the connection between two-dimensional $\mathcal{N}=2 \mathrm{SYM}$ theory and a cohomological field theory used in section 4 .

\section{Matrix Strings}

In this section we review some basic properties of the IIA and IIB matrix string theories, which are derived from the M(atrix) theory by considering toroidal compactification on $S^{1}$ and on $T^{2}$ respectively. In addition, we give an argument that two spinors of the same space-time chirality emerge in the IIB matrix string theory, which has not been found in the literatures.

We start with the $\mathrm{M}$ (atrix) theory [4], whose action has the same form as low energy effective action of $N$ D0-branes, that is one-dimensional $U(N)$ SYM theory with 16 supercharges. The BFSS conjecture is that this action exactly describes M-theory in the decompactified limit $R_{11} \rightarrow \infty$ by going to the infinite momentum frame. The infinite momentum frame means infinite amount of boosting along the 11th direction, i.e. momentum of the 11th direction becomes

$$
p_{11}=\frac{N}{R_{11}} \rightarrow \infty .
$$

At the same time we must take the limit $N, R_{11} \rightarrow \infty$, in order that the resulting theory represents the strongly coupled limit of type IIA superstring theory. 


\subsection{IIA Matrix String Theory}

Let us consider the compactification of one of the transverse directions, say the 9th, to a circle of the circumference $L$, denoted by $S^{1}(L)$. Since in the M(atrix) theory we consider the limit that the 11th direction is decompactified, we have ten-dimensional type IIA string theory with the string length

$$
l_{s}^{2}=\frac{2 \pi l_{11}^{3}}{L} .
$$

According to the prescription of Taylor [5], this IIA string theory is described by twodimensional $\mathcal{N}=8 U(N)$ SYM theory, which is a dimensional reduction of ten-dimensional $\mathcal{N}=1$ SYM theory [6],

$$
\begin{aligned}
S_{\mathrm{IIA}-\mathrm{MS}}= & \frac{1}{g^{2}} \int d t \int_{0}^{R} d \sigma \operatorname{tr}\left[-\frac{1}{4} F_{\mu \nu} F^{\mu \nu}-\frac{1}{2}\left(D_{\mu} X^{I}\right)^{2}+i \theta^{T}\left(D_{t}+\Gamma^{9} D_{\sigma}\right) \theta\right. \\
& \left.+\frac{1}{4}\left[X^{I}, X^{J}\right]^{2}+\theta^{T} \Gamma^{I}\left[X_{I}, \theta\right]\right] .
\end{aligned}
$$

Here, $F_{\mu \nu}=\partial_{\mu} A_{\nu}-\partial_{\nu} A_{\mu}-i\left[A_{\mu}, A_{\nu}\right]$ is a field strength made from two-dimensional gauge field $A_{\mu}, X^{I}(I=1, \cdots, 8)$ are Higgs fields and $\theta^{\alpha}(\alpha=1, \cdots, 16)$ are fermions. We use the convention that the $\Gamma$-matrices are $16 \times 16$ real symmetric matrices satisfying

$$
\left\{\Gamma^{I}, \Gamma^{J}\right\}=2 \delta^{I J}, \quad \Gamma^{9}=\Gamma^{1} \cdots \Gamma^{8} .
$$

$\theta^{\alpha}$ 's are decomposed into the spinor and conjugate spinor representations $\left(\boldsymbol{8}_{\mathbf{s}} \oplus \mathbf{8}_{\mathbf{c}}\right)$ of the rotational group in the transverse directions $S O(8)$, which are characterized by the eigenvalues of $\Gamma^{9}$, so called chirality:

$$
\theta^{\alpha}=\theta_{+}^{\alpha}+\theta_{-}^{\alpha}, \quad \Gamma^{9} \theta_{ \pm}^{\alpha}= \pm \theta_{ \pm}^{\alpha},
$$

and $X^{I}$ 's transform as the vector $\mathbf{8}_{\mathbf{v}}$. The spatial coordinate $\sigma$, which arises as performing the compactification, takes a value on the circle dual to $S^{1}(L): 0 \leq \sigma \leq R$, wheref

$$
R=(2 \pi)^{2} \frac{l_{s}^{2}}{L}
$$

The coupling constant of the SYM theory $g$ is related to the string coupling $g_{s}$ as

$$
g^{2}=\frac{2 \pi}{\left(R g_{s}\right)^{2}}
$$

Weakly coupled strings are recovered by considering the limit $g_{s} \rightarrow 0$ or equivalently the infra-red limit of the SYM theory [6]. In this situation, the theory is described by the eigenvalues of the simultaneously diagonalizable configurations of $X^{I}$ and $\theta^{\alpha}$ :

$$
\tilde{X}^{I}=\operatorname{diag}\left(x_{1}^{I}, \cdots, x_{N}^{I}\right), \quad \tilde{\theta^{\alpha}}=\operatorname{diag}\left(\theta_{1}^{\alpha}, \cdots, \theta_{N}^{\alpha}\right)
$$

2 The relation between parameters in the matrix string theory and those in SYM theory, eqs. (7) and (8) in the IIA case as well as eqs. (18), (19) and (20) in the IIB case, can be derived either by tracing the procedure by Taylor [5] or by employing another argument in ref. [15]. 
where

$$
X^{I}=V \tilde{X}^{I} V^{\dagger}, \quad \theta^{\alpha}=V \tilde{\theta^{\alpha}} V^{\dagger} .
$$

The angular variables $V \in U(N)$ and nontrivial configurations of the gauge field yielding non-zero curvature $F_{\mu \nu}$ are energetically decoupled from the theory in the infra-red limit, and the action (蛋) reduces to the action of multiple Green-Schwarz superstrings in the light-cone gauge. Then the gauge field can take the pure gauge configuration

$$
A_{\mu}=i V \partial_{\mu} V^{\dagger}
$$

Also, the $x_{i}^{I}$ and $\theta_{i}^{\alpha}$ can represent strings of various lengths by considering the multi-valued configuration:

$$
\begin{aligned}
& \tilde{X}^{I}(t, \sigma+R)=g^{\dagger} \tilde{X^{I}}(t, \sigma) g, \quad \tilde{\theta^{\alpha}}(t, \sigma+R)=g^{\dagger} \tilde{\theta}^{\alpha}(t, \sigma) g, \\
& V(t, \sigma+R)=V(t, \sigma) g,
\end{aligned}
$$

where $g$ is an element of the Weyl group of $U(N)$, i.e. the permutation group $S_{N}$, which permutes the $N$ eigenvalues. In going around the $\sigma$-direction, the eigenvalues are interchanged by the action of $g$ in eqs. (12), and as a result they form cycles of various lengths corresponding to permutation cycles in $g$. Each cycle is interpreted as a single closed string, and thus for $g \in S_{N}$ consisting of $N_{n} n$-cycles (satisfying $N=\sum_{n} n N_{n}$ ) one has $N_{n}$ strings with length $n$.

Note that the total matrices $X^{I}, \theta^{\alpha}$ in eqs. (10) and the gauge field $A_{\mu}$, which appear in the SYM theory, remain single-valued although we consider the case that the variables of string coordinates $\tilde{X}^{I}$ and $\tilde{\theta}^{\alpha}$ are multi-valued as in eqs. (12), which is argued in ref. [16]. For the simplest $N=2$ case, the multi-valued configuration is represented by

$$
\tilde{X}^{I}=\left(\begin{array}{cc}
x_{1}^{I} & 0 \\
0 & x_{2}^{I}
\end{array}\right), \quad \tilde{\theta^{\alpha}}=\left(\begin{array}{cc}
\theta_{1}^{\alpha} & 0 \\
0 & \theta_{2}^{\alpha}
\end{array}\right), \quad V=\frac{1}{\sqrt{2}}\left(\begin{array}{cc}
e^{i \pi \sigma / R} & -e^{i \pi \sigma / R} \\
1 & 1
\end{array}\right),
$$

where

$$
x_{1}^{I}(t, \sigma+R)=x_{2}^{I}(t, \sigma), \quad x_{2}^{I}(t, \sigma+R)=x_{1}^{I}(t, \sigma),
$$

and $\theta_{i}^{\alpha}$ satisfies the same boundary condition. The matrices $X^{I}, \theta^{\alpha}$ and $A_{\mu}$ are single-valued. In particular, the gauge field is

$$
A_{t}=0, \quad A_{\sigma}=\frac{1}{2}\left(\begin{array}{ll}
1 & 0 \\
0 & 0
\end{array}\right),
$$

which cannot be eliminated by any single-valued gauge transformation.

As discussed in [6], relaxing the strict limit $g_{s}=0$, the number of the strings are no longer conserved. We can interpret this as a result of string interactions (splitting or joining of one or two strings) which occur when the two sets of eigenvalues $\left\{x_{i}^{I}\right\}_{I=1, \cdots, 8}$ and $\left\{x_{j}^{I}\right\}_{I=1, \cdots, 8}$ coincide. Whenever the interaction occurs, the boundary condition of $\tilde{X}^{I}, \tilde{\theta^{\alpha}}$ and $V$ is changed, i.e. a branch point appears on a cylinder coordinated by $t$ and $\sigma$. We 
should remark that as discussed in [17], in spite of this singular configuration of the diagonal variables, for a neighborhood of the branch point the corresponding total matrices exist as a smooth and single-valued configuration of the SYM theory. In the section 4 of ref. [17] such a configuration is constructed in the $N=2$ case, which is given by a solution of the dimensionally reduced version of the self-dual equation of four-dimensional Yang-Mills theory to two dimensions:

$$
\begin{aligned}
& {\left[X_{1}, X_{2}\right]=\frac{i}{g^{2}} F_{t \sigma}} \\
& D_{t} X_{1}=-D_{\sigma} X_{2} \\
& D_{t} X_{2}=D_{\sigma} X_{1}
\end{aligned}
$$

and the all other fields are set to zero. Compared to the configuration in the strict limit $\left(g^{2}=\infty\right)$ that is $\left[X_{1}, X_{2}\right]=0$, the $O\left(1 / g^{2}\right)$-correction in eq. (15) works well to make the configuration in the SYM theory smooth

\subsection{IIB Matrix String Theory}

When compactifying IIA string theory to a circle and taking T-dual to the circle, we have IIB string theory on $S^{1}$. In the limit of shrinking the circle of the IIA theory, the $S^{1}$ of the IIB theory is decompactified, thus we have IIB string theory in ten dimensions. From the view point of M-theory, IIA theory on $S^{1}$ means M-theory on $T^{2}$. We denote the size of the two-torus by $L_{1}$ and $L_{2}$, i.e. $T^{2}=S^{1}\left(L_{1}\right) \times S^{1}\left(L_{2}\right)$. Since there is no distinctive meaning between the two $S^{1}$ 's, now we have two ways to obtain IIB theory corresponding to shrinking either $L_{1}$ or $L_{2}$. From the analysis based on low energy effective theories, two IIB theories we obtain as the result are believed to be equivalent and connected by S-duality [19, 20]. In this way, the M-theory perspective yields a geometrical interpretation to the S-duality in type IIB string theory.

Let us consider this operation in the M(atrix) theory. Then we obtain three-dimensional $\mathcal{N}=8 U(N)$ SYM theory, which is dimensionally reduced from the ten-dimensional theory, as a matrix model corresponding to IIB theory on $S^{1}$ [7, \&]:

$$
\begin{aligned}
S_{\mathrm{IIB}-\mathrm{MS}}= & \frac{1}{g^{2}} \int d t \int_{0}^{R_{1}} d \sigma_{1} \int_{0}^{R_{2}} d \sigma_{2} \operatorname{tr}\left[-\frac{1}{4} F_{\mu \nu} F^{\mu \nu}-\frac{1}{2}\left(D_{\mu} X^{I}\right)^{2}\right. \\
& \left.+i \theta^{T}\left(D_{t}+\Gamma^{8} D_{\sigma_{1}}+\Gamma^{9} D_{\sigma_{2}}\right) \theta+\frac{1}{4}\left[X^{I}, X^{J}\right]^{2}+\theta^{T} \Gamma^{I}\left[X_{I}, \theta\right]\right] .
\end{aligned}
$$

Here we compactified the 8th and 9th transverse directions to the above mentioned (rectangular) two-torus. The size of the spatial directions $\left(\sigma_{1}\right.$ and $\left.\sigma_{2}\right)$ which the SYM theory is defined on is related by T-duality to the two-torus,

$$
R_{i}=(2 \pi)^{2} \frac{l_{s}^{2}}{L_{i}} \quad(i=1,2)
$$

\footnotetext{
${ }^{3}$ An extension of this argument in the case of general $N$ is discussed in refs. [18]. I thank L. Bonora for informing me of those literatures.
} 
and the SYM coupling are given by

$$
g^{2}=\frac{R_{1} R_{2} R_{11}}{(2 \pi)^{2} l_{s}^{4}}
$$

It is remarkable that the string coupling is given by a ratio of the two lengths of the torus:

$$
g_{s}=\frac{R_{1}}{R_{2}}=\frac{L_{2}}{L_{1}}
$$

which gives the geometrical understanding of S-duality also in the matrix string level. In the vanishing torus limit $L_{1}, L_{2} \rightarrow 0$, a new dimension opens up and becomes decompactified, so this limit with the ratio $L_{2} / L_{1}$ fixed is considered to give type IIB theory of the coupling $g_{s}$ determined by eq. (20) [8, 15]. Note that the abelian part of the field strength $F_{\mu \nu}$ made from three-dimensional gauge field $A_{\mu}$ reduces to a single scalar field via duality transformation. Although the manifest symmetry of the action (17) is $S O(7)$, it is considered that this scalar and the seven Higgs fields $X^{I}(I=1, \cdots, 7)$ together belong to $\mathbf{8}_{\mathbf{v}}$ in $S O(8)$ in the ten-dimensional IIB limit

$$
R_{1} R_{2} \rightarrow \infty, \quad g_{s}: \text { fixed }
$$

by the argument for BPS states [15] and by the analysis of the moduli space of the threedimensional SYM theory [7, 11]. The fermions $\theta^{\alpha}(\alpha=1, \cdots, 16)$ are to represent two space-time spinors of the same chirality in the IIB theory. In fact, after decomposing by the eigenvalues of $\Gamma^{9}$ as in eq. (6), we put

$$
\psi_{+}^{\alpha}=\Gamma^{8} \theta_{-}^{\alpha}
$$

Then fermion part of the lagrangian density takes the form

$$
\begin{aligned}
& \operatorname{tr}\left[i \theta_{+}^{T}\left(D_{t}+D_{\sigma_{2}}\right) \theta_{+}+i \psi_{+}^{T}\left(D_{t}-D_{\sigma_{2}}\right) \psi_{+}+i \theta_{+}^{T} D_{\sigma_{1}} \psi_{+}+i \psi_{+}^{T} D_{\sigma_{1}} \theta_{+}\right. \\
& \left.+\theta_{+}^{T} \Gamma^{I} \Gamma^{8}\left[X^{I}, \psi_{+}\right]-\psi_{+}^{T} \Gamma^{I} \Gamma^{8}\left[X^{I}, \theta_{+}\right]\right] .
\end{aligned}
$$

Both of $\theta_{+}$and $\psi_{+}$are spinors with respect to the manifest symmetry $S O(7)$ of the same eigenvalue $(+1)$ of $\Gamma^{9}$. In the weakly coupled limit $g_{s} \ll 1$, which means $R_{1} \ll R_{2}$, nonzero modes of $\partial_{\sigma_{1}}$ energetically decouple, and $\theta_{+}$and $\psi_{+}$represent the two $\mathbf{8}_{\mathbf{s}}$ spinors in IIB theory. Also, in the strongly coupled limit $R_{2} \ll R_{1}$, it turns out that $\xi_{+}=\left(\theta_{+}+\psi_{+}\right) / \sqrt{2}$ and $\eta_{+}=\left(\theta_{+}-\psi_{+}\right) / \sqrt{2}$ become spinors desired in IIB theoryf. In this way, we can see that in both of the two limits related via S-duality chiral spinors are correctly reproduced in the IIB matrix string theory.

\footnotetext{
${ }^{4}$ In IIA matrix string theory, by the same replacement (22), we seem to have chiral IIB theory, but it is not correct. The IIA matrix string theory has the manifest symmetry $S O(8)$. Note that $\psi_{+}$is not a spinor under the $S O(8)$ due to the $\Gamma^{8}$ factor in eq. (22).
} 


\section{Partition Function of four-dimensional $\mathcal{N}=4 S U(N)$ Super Yang-Mills Theory}

Here we calculate the partition function of $\mathcal{N}=4 S U(N)$ SYM theory on four-torus $T^{4}$ by mapping the theory to a cohomological field theory. The dimensional reduced version of this argument is used in later computations of the partition function of IIA and IIB matrix string theories.

$\mathcal{N}=4 S U(N)$ SYM theory on a flat four-dimensional space-time is given by the following lagrangian density defined on the $\mathcal{N}=1$ superspace $(x, \theta, \bar{\theta})$ :

$$
\begin{aligned}
\mathcal{L}_{\mathcal{N}=4}= & \frac{1}{16 g^{2}} \operatorname{tr}\left(\left.W^{\alpha} W_{\alpha}\right|_{\theta \theta}+\left.\bar{W}_{\dot{\alpha}} \bar{W}^{\dot{\alpha}}\right|_{\bar{\theta} \bar{\theta}}\right) \\
& +\left.\frac{1}{g^{2}} \operatorname{tr}\left(\Phi_{1}^{\dagger} e^{V} \Phi_{1}+\Phi_{2}^{\dagger} e^{V} \Phi_{2}+\Phi_{3}^{\dagger} e^{V} \Phi_{3}\right)\right|_{\theta \theta \bar{\theta} \bar{\theta}} \\
& +\frac{1}{\sqrt{2} g^{2}} \operatorname{tr}\left(\left.\Phi_{1}\left[\Phi_{2}, \Phi_{3}\right]\right|_{\theta \theta}+\left.\Phi_{1}^{\dagger}\left[\Phi_{3}^{\dagger}, \Phi_{2}^{\dagger}\right]\right|_{\bar{\theta} \bar{\theta}}\right) .
\end{aligned}
$$

In this section, we use the notation in ref. [21]. The vector superfield $V$ represents a multiplet containing a gauge field $A_{m}$ and a complex two-component gauge fermion $\lambda$, and the chiral superfield $\Phi_{s}(s=1,2,3)$ contains a multiplet of a complex Higgs scalar $B_{s}$ and a Higgsino $\psi_{s}$. All the fields belong to the adjoint representation of $S U(N)$, "tr" denotes the trace in the fundamental representation. The theory has the internal symmetry group $S U(4)_{I}$, under which the fermions ( $\lambda$ and $\psi_{s}$ 's) transform together as $\mathbf{4}$ and $B_{s}$ 's as $\mathbf{6}$. Also, the supercharges $Q_{\alpha}^{v}, \bar{Q}_{v \dot{\alpha}}$ as $4, \overline{4}$ respectively, where $v(=1, \cdots, 4)$ is the $S U(4)_{I}$ index and $\alpha, \dot{\alpha}$ are Lorentz indices belonging to $S U(2)_{L}, S U(2)_{R}$.

In terms of the component fields, the lagrangian takes the form

$$
\begin{aligned}
& \mathcal{L}_{\mathcal{N}=4}= \frac{1}{g^{2}} \operatorname{tr}\left[-\frac{1}{4} F^{m n} F_{m n}-i \bar{\lambda} \bar{\sigma}^{m} D_{m} \lambda \frac{1}{2} D^{2}\right] \\
&+\frac{1}{g^{2}} \sum_{s=1}^{3} \operatorname{tr}\left[F_{s}^{\dagger} F_{s}-\left(D^{m} B_{s}\right)^{\dagger}\left(D_{m} B_{s}\right)-i \bar{\psi}_{s} \bar{\sigma}^{m} D_{m} \psi_{s}\right. \\
&\left.\quad-\frac{i}{\sqrt{2}} B_{s}^{\dagger}\left[\lambda, \psi_{s}\right]+\frac{i}{\sqrt{2}} B_{s}\left[\bar{\lambda}, \bar{\psi}_{s}\right]+\frac{1}{2} D\left[B_{s}, B_{s}^{\dagger}\right]\right] \\
&+\frac{1}{\sqrt{2} g^{2}} \operatorname{tr}\left(F_{1}\left[B_{2}, B_{3}\right]+F_{2}\left[B_{3}, B_{1}\right]+F_{3}\left[B_{1}, B_{2}\right]\right. \\
&-B_{1}\left[\psi_{2}, \psi_{3}\right]-B_{2}\left[\psi_{3}, \psi_{1}\right]-B_{3}\left[\psi_{1}, \psi_{2}\right] \\
&+ \text { h.c. }),
\end{aligned}
$$

where $F_{s}$ and $D$ are auxiliary fields appearing in $\Phi_{s}$ and $V$. 


\subsection{Twisting of $\mathcal{N}=4$ Super Yang-Mills Theory}

The twisting procedure, which was first introduced by Witten [22], gives a systematic tool for constructing a cohomological field theory from the original physical theory. Here we briefly explain a twisting procedure of $N=4$ SYM theory adopted by Vafa and Witten [23].

First, we consider a general four-manifold on which the $\mathcal{N}=4$ SYM theory is defined. The holonomy group of this manifold is $S O(4)=S U(2)_{L} \times S U(2)_{R}$, that is a gauged symmetry with the spin connection being the (external) gauge field. Adding the global $S U(4)_{I}$, we concentrate the symmetry group of the theory

$$
H=S U(2)_{L} \times S U(2)_{R} \times S U(4)_{I} .
$$

The twisting by Vafa and Witten is performed as follows. Consider the subgroup of the internal symmetry group $S U(4)_{I}$ :

$$
S U(4)_{I} \supset S O(4)=S U(2)_{F} \times S U(2)_{F^{\prime}},
$$

then replace the action of $S U(2)_{L}$ by the diagonal sum $S U(2)_{L}^{\prime}=S U(2)_{L} \oplus S U(2)_{F^{\prime}}$. Thus the twisted symmetry group becomes

$$
H^{\prime}=S U(2)_{L}^{\prime} \times S U(2)_{R} \times S U(2)_{F} .
$$

Under $H^{\prime}$, the supercharges split up as

$$
\begin{aligned}
& Q_{\alpha}^{v}=Q_{\alpha}^{i j} \rightarrow Q_{\alpha}^{i \beta}=\left\{\begin{array}{l}
Q_{\alpha}^{i \alpha} \equiv Q^{i} \\
\frac{1}{2} \varepsilon_{\gamma(\beta} Q_{\alpha)}^{i \gamma} \equiv Q_{\alpha \beta}^{i}
\end{array}\right. \\
& \bar{Q}_{v \dot{\alpha}}=\bar{Q}_{i j \dot{\alpha}} \rightarrow \bar{Q}_{i \beta \dot{\alpha}},
\end{aligned}
$$

where $i$ and $j$ are $S U(2)_{F}$ and $S U(2)_{F^{\prime}}$ indices respectively, also $j$ is converted to $\beta$ by the twisting. Here we have the two scalar supercharges $Q^{i}(i=1,2)$, which are nilpotent

$$
\left\{Q^{i}, Q^{j}\right\}=0
$$

as seen from the supersymmetry algebra of the original supercharges

$$
\begin{aligned}
& \left\{Q_{\alpha}^{v}, \bar{Q}_{w \dot{\alpha}}\right\}=2 \sigma_{\alpha \dot{\alpha}}^{m} \delta_{w}^{v} P_{m}, \\
& \left\{Q_{\alpha}^{v}, Q_{\beta}^{w}\right\}=\left\{\bar{Q}_{v \dot{\alpha}}, \bar{Q}_{w \dot{\beta}}\right\}=0 .
\end{aligned}
$$

We can construct a cohomological field theory based on each $Q^{i}$ 's. The $Q^{i}$ s are related to the original supercharges as

$$
\begin{aligned}
& Q^{1}=Q_{1}^{11}+Q_{2}^{12}=Q_{\alpha=1}^{v=1}+Q_{\alpha=2}^{v=2}, \\
& Q^{2}=Q_{1}^{21}+Q_{2}^{22}=Q_{\alpha=1}^{v=3}+Q_{\alpha=2}^{v=4}
\end{aligned}
$$

Next, in the case of four-dimensional Kähler manifolds, the number of the nilpotent scalar supercharges become doubled as we will see ${ }^{\text {}}$. A four-dimensional Kähler manifold

\footnotetext{
${ }^{5}$ This property was explored first by Witten [25] in the case of $\mathcal{N}=2$ SYM theory.
} 
has the reduced holonomy group $U(1)_{L} \times S U(2)_{R}$, where $U(1)_{L}$ is a certain subgroup of $S U(2)_{L}$. In this case, twisting is done by replacing the $U(1)_{L}$ by the diagonal sum $U(1)_{L}^{\prime}=$ $U(1)_{L} \oplus U(1)_{F^{\prime}}$. Here, $U(1)_{F^{\prime}}$ is a subgroup of $S U(2)_{F^{\prime}}$. We assign the $U(1)_{L}$ charge +1 to $Q_{\alpha=1}^{v}$ and -1 to $Q_{\alpha=2}^{v}$, and give a similar assignment of the $U(1)_{F^{\prime}}$ charge $(+1$ to the $j=1$ index and -1 to the $j=2$ ). Then by twisting, the four $Q_{\alpha}^{v}$ 's in eqs. (28) are to have zero $U(1)_{L}^{\prime}$ charge, that is, they all become scalar supercharges, since $Q_{1}^{i 1}=Q_{12}^{i}$ and $Q_{2}^{i 2}=-Q_{21}^{i}$. It is clear that they are nilpotent. Note that one of the four charges is a (chiral) generator of the manifest $\mathcal{N}=1$ supersymmetry in eq. (24). We will use that charge, denoted by $Q$, to construct a cohomological field theory in the next subsection.

Since the twisting changes the coupling of the fields with the spin connection [24], for a general four-manifold the twisted theory differs from the original theory. However, on a manifold with the flat metric $\left(T^{4}\right)$ or more generally on a hyper-Kähler manifold for which the twisting is a trivial operation owing to its holonomy group $S U(2)_{R}$, the twisted theory coincides with the original theory. Now we will investigate the $T^{4}$ case.

\subsection{Partition function of $\mathcal{N}=4 \mathrm{SYM}$ on $T^{4}$}

The $\mathcal{N}=4 S U(N)$ SYM theory defined on $T^{4}$ can be seen as a cohomological field theory by (trivial) twisting, because of the reason mentioned above. In the action $S_{\mathcal{N}=4}$ (24), since $Q$ acts as $\frac{\partial}{\partial \theta^{\alpha}}=\int d \theta^{\alpha}$ (up to total derivative terms) for suitable $\alpha$, the term with $\left.\right|_{\theta \theta}$ are written in the $Q$-exact form $\{Q, \cdots\}$. Also, the term $\left.\operatorname{tr} \bar{W}_{\dot{\alpha}} \bar{W}^{\dot{\alpha}}\right|_{\bar{\theta} \bar{\theta}}$ is equal to $\left.\operatorname{tr} W^{\alpha} W_{\alpha}\right|_{\theta \theta}$ modulo the total derivative term $\operatorname{tr} F \wedge F$, so it is of the $Q$-exact form. Here we consider the periodic boundary condition case on $T^{4}$, or equivalently the sector of zero 't Hooft discrete magnetic flux, those total derivative terms can be discarded. The term $\left.\operatorname{tr} \Phi_{1}^{\dagger}\left[\Phi_{3}^{\dagger}, \Phi_{2}^{\dagger}\right]\right|_{\bar{\theta} \bar{\theta}}$ can not be written as the $Q$-exact form, but it is $Q$-invariant because $Q$ is a part of the manifest $\mathcal{N}=1$ supersymmetric transformation. Thus the action can be written as

$$
S_{\mathcal{N}=4}=\{Q, \cdots\}+\mathcal{O}^{(0)},
$$

where

$$
\mathcal{O}^{(0)}=\left.\int d^{4} x \frac{1}{\sqrt{2} g^{2}} \operatorname{tr} \Phi_{1}^{\dagger}\left[\Phi_{3}^{\dagger}, \Phi_{2}^{\dagger}\right]\right|_{\bar{\theta} \bar{\theta}}
$$

is a $Q$-invariant operator and the superscript 0 stands for the $U(1)$-charge defined by the rotation

$$
\begin{aligned}
\Phi_{s}(\theta, \bar{\theta}, x) & \rightarrow e^{\frac{2}{3} i \beta} \Phi_{s}\left(e^{-i \beta} \theta, e^{i \beta} \bar{\theta}, x\right) \\
W^{\alpha}(\theta, \bar{\theta}, x) & \rightarrow e^{i \beta} W^{\alpha}\left(e^{-i \beta} \theta, e^{i \beta} \bar{\theta}, x\right)
\end{aligned}
$$

with $\beta$ being a real parameter. The charge of the component fields can be read off from eqs.

(31) as $-\frac{2}{3},+\frac{1}{3},+\frac{4}{3}, 0,-1,0$ for $B_{s}, \psi_{s}, F_{s}, A_{m}, \lambda, D$, respectively. Note that the action has the symmetry under this rotation.

Now the problem can be made more tractable by considering the following mass perturbation

$$
\Delta S=\int d^{4} x \frac{1}{\sqrt{2} g^{2}}\left(-\frac{m}{2}\right) \operatorname{tr}\left[\left.\left(\Phi_{1}^{2}+\Phi_{2}^{2}+\Phi_{3}^{2}\right)\right|_{\theta \theta}+\left.\left(\Phi_{1}^{\dagger 2}+\Phi_{2}^{\dagger 2}+\Phi_{3}^{\dagger 2}\right)\right|_{\bar{\theta} \bar{\theta}}\right]
$$


Using the same argument as above, the first term is written in the form $\{Q, \cdots\}$, and the second term is a $Q$-invariant operator with the $U(1)$-charge $-\frac{2}{3}$ denoted by $\mathcal{O}^{(-2 / 3)}$. In cohomological field theories, the perturbation by the $Q$-exact operators does not alter the theory, and the partition function is invariant under the perturbation of $\mathcal{O}^{(-2 / 3)}$ if the $U(1)$ symmetry remains left even in the quantum level. In fact, it is so since there is no anomaly in $\mathcal{N}=4$ theory. Thus we can obtain the answer by computing the partition function of the mass perturbed system.

Here, it is remarked that the $U(1)$-symmetry is different from the ghost number symmetry usually used in cohomological field theories. In fact, it is seen that in the cohomological field theory made from $\mathcal{N}=4 \mathrm{SYM}$ theory the ghost number symmetry corresponds to the following rotation [23, 26]

$$
\begin{aligned}
\Phi_{1}(\theta, \bar{\theta}, x) & \rightarrow \Phi_{1}\left(e^{-i \beta} \theta, e^{i \beta}, x\right) \\
\Phi_{2}(\theta, \bar{\theta}, x) & \rightarrow \Phi_{2}\left(e^{-i \beta} \theta, e^{i \beta}, x\right) \\
\Phi_{3}(\theta, \bar{\theta}, x) & \rightarrow e^{2 i \beta} \Phi_{3}\left(e^{-i \beta} \theta, e^{i \beta}, x\right) \\
W^{\alpha}(\theta, \bar{\theta}, x) & \rightarrow e^{i \beta} W^{\alpha}\left(e^{-i \beta} \theta, e^{i \beta} \bar{\theta}, x\right) .
\end{aligned}
$$

Vafa and Witten [23] discussed the invariance of the partition function under the mass perturbation based on this ghost number symmetry. Our argument presents another proof of the invariance.

We should remark that cohomological field theories have an important feature that the contribution of the path integration localizes in configurations of the classical vacua. In the mass perturbed system, the classical vacua are given by the solutions of these equations:

$$
\begin{aligned}
& {\left[B_{1}, B_{2}\right]=m B_{3},} \\
& {\left[B_{2}, B_{3}\right]=m B_{1},} \\
& {\left[B_{3}, B_{1}\right]=m B_{2}}
\end{aligned}
$$

with

$$
\sum_{s=1}^{3}\left[B_{s}, B_{s}^{\dagger}\right]=0 .
$$

A special property of the perturbation is that three equations in (33) have the form of $S U(2)$ algebra. So, the solution $\frac{i}{m} B_{s}$ is given by the generator of $N$-dimensional representation of $S U(2)$. At the same time, this solution satisfies eq. (34) also, because $B_{s}$ 's are anti-hermitian. Since we must consider the various $N$-dimensional representations, both of reducible and irreducible, in general the solution takes the form

$$
\frac{i}{m} B_{s}=\left[\begin{array}{cccc}
L_{s}^{\left(a_{1}\right)} & & & \\
& L_{s}^{\left(a_{2}\right)} & & \\
& & \ddots & \\
& & & L_{s}^{\left(a_{l}\right)}
\end{array}\right]
$$


where $L_{s}^{(a)}$ is a generator of $a$-dimensional irreducible representation of $S U(2)$, and $a_{1}+a_{2}+$ $\cdots+a_{l}=N$. We remark that all the solutions of the form (35) do not contribute to the partition function, but only the $a_{1}=a_{2}=\cdots=a_{l}$ case does. In fact, considering the $l=2$ case, it is easy to see that the unbroken gauge group of the solution (35) contains a following $U(1)$-generator

$$
\left[\begin{array}{cc}
e_{1} \mathbf{1}_{a_{1}} & 0 \\
0 & e_{2} \mathbf{1}_{a_{2}}
\end{array}\right],
$$

where $e_{1}$ and $e_{2}$ are real parameters satisfying $e_{1} a_{1}+e_{2} a_{2}=0$. Let us consider the $a_{1} \neq a_{2}$ case. The interactions are all in the form of commutators, so this $U(1)$ mode is free and massless, which implies that there exists a fermion zero-mode corresponding to the $U(1)$. Thus its contribution to the partition function vanishes. However, when $a_{1}=a_{2}$, the $B_{s}$ is written as

$$
\frac{i}{m} B_{s}=\mathbf{1}_{2} \otimes L_{s}^{(a)}
$$

where we put $a_{1}=a_{2} \equiv a$. Then it is noted that the unbroken gauge group is enhanced to $S U(2) \otimes \mathbf{Z}_{a}$. This mode is not free and thus can give a certain contribution to the partition function, which amounts to a $\mathcal{N}=1 S U(2) \otimes \mathbf{Z}_{a}$ SYM theory. For the case of generic $l$, a similar argument goes on and it can be seen that the vacuum which can contribute to the partition function is a $\mathcal{N}=1 S U(l) \otimes \mathbf{Z}_{a}$ SYM theory with $a l=N$. In the consequence the partition function of the $\mathcal{N}=4$ theory is represented as a sum of the partition function of the various $\mathcal{N}=1$ theories:

$$
Z_{S U(N)}^{D=4, \mathcal{N}=4}\left(T^{4}\right)=\sum_{a l=N} Z_{S U(l) \otimes \mathbf{Z}_{a}}^{D=4, \mathcal{N}=1}\left(T^{4}\right) .
$$

Further, since the $\mathbf{Z}_{a}$ factor in the gauge group $S U(l) \otimes \mathbf{Z}_{a}$ implies a summation of the flat $\mathbf{Z}_{a}$ bundle, it yields a factor $a^{4-1}=a^{3}$, where the power 4 comes from summing up the flat bundle for each $A_{m}$ 's and the $(-1)$ from dividing by the gauge group $\mathbf{Z}_{a}$. Thus we have

$$
Z_{S U(N)}^{D=4, \mathcal{N}=4}\left(T^{4}\right)=\sum_{a l=N} a^{3} Z_{S U(l)}^{D=4, \mathcal{N}=1}\left(T^{4}\right) .
$$

Here we should note that the partition function of the $\mathcal{N}=1$ theory in the right hand side is given by its Witten index. In the Hamiltonian formalism, the partition function is written as

$$
\operatorname{Tr}(-1)^{F} e^{-\beta H}
$$

where the $(-1)^{F}$-factor is included in order to impose the periodic boundary condition on the fermion fields. Recall that there is no Higgs field in the $\mathcal{N}=1$ theory, which implies that there exists no continuous zero-mode in the theory on $T^{4}$. Spectra appearing in the theory are all discrete. Then in eq. (40), the contribution of every supersymmetric pair with non-zero energy is precisely cancelled, and only the zero-energy states can contribute. So eq. (40) is independent of $\beta$, and it coincides with the Witten index

$$
I \equiv \lim _{\beta \rightarrow \infty} \operatorname{Tr}(-1)^{F} e^{-\beta H},
$$


whose value of the $\mathcal{N}=1 S U(l)$ SYM theory is known to be $l$ [27. Plugging the above formulas, we obtain the answer

$$
Z_{S U(N)}^{D=4, \mathcal{N}=4}\left(T^{4}\right)=\sum_{a l=N} a^{3} l=N \sum_{a \mid N} a^{2}
$$

where the summation of $a$ in the right hand side is taken over the divisors of $N$.

Also, for the gauge group $S U(N) / \mathbf{Z}_{N}$ we can obtain the partition function of the sector of zero 't Hooft magnetic flux applying the above argument to the $\mathbf{Z}_{N}$-factor

$$
Z_{S U(N) / \mathbf{Z}_{N}}^{D=4, \mathcal{N}=4}\left(T^{4}\right)=\frac{1}{N^{3}} Z_{S U(N)}^{D=4, \mathcal{N}=4}\left(T^{4}\right)=\sum_{a \mid N} \frac{1}{a^{2}} .
$$

\section{IIA Matrix String Partition Function}

Here we calculate the partition function of IIA matrix string theory and compare the result of Kostov and Vanhove [13] which has been derived in the case of the strong coupling limit $g \rightarrow \infty$. We have seen in section 2 that the SYM fields in periodic boundary condition can reproduce the second quantized superstrings with interactions. Thus, considering the twodimensional SYM theory with periodic boundary condition as the IIA matrix string theory, we evaluate the partition function of this theory.

Now we have to take care of the $U(1)$ part of the gauge group $U(N)$ before doing the calculation. We consider the gauge group $U(N)$ in the factorized form

$$
U(N)=U(1) \times\left(S U(N) / \mathbf{Z}_{N}\right) .
$$

The meaning of the $U(1)$ part in the IIA matrix string theory, whose field contents are $\left(A_{\mu}, X, \theta\right)$, is as follows. The $U(1)$ part of $X$ and $\theta$ represents the center of mass coordinates of the strings in transverse directions. We fix it from the translational invariance. On the other hand, the $U(1)$ part of $A_{\mu}$ is related to the number of D-particles, so we have to take into account this. More precisely, as is discussed in [6], the $U(1)$ electric flux corresponds to the number of D-particles:

$$
q=\frac{1}{2 \pi} \int_{0}^{R} d \sigma E^{U(1)} \in \mathbf{Z} .
$$

Corresponding to eq. (44), the gauge field $A_{\mu}$ is decomposed as

$$
A_{\mu}=A_{\mu}^{U(1)} T^{U(1)}+A_{\mu}^{a} T^{a}
$$

where $T^{U(1)}=\frac{1}{N} \mathbf{1}_{N}$, and $T^{a}\left(a=1, \cdots, N^{2}-1\right)$ is a generator of $S U(N)$. Since the interaction in the SYM theory appears in the form of commutators, the $U(1)$ gauge part decouples and thus the partition function of the IIA matrix string theory becomes

$$
Z_{\text {IIA-MS }}=\left(\int \frac{\mathcal{D} A_{\mu}^{U(1)}}{\operatorname{Vol}(U(1))} e^{-S^{U(1)}}\right) Z_{S U(N) / \mathbf{Z}_{N}}^{D=2, \mathcal{N}=8}\left(T^{2}\right),
$$


where the Wick rotation was performed. The two-torus, where the SYM theory is defined on, is a rectangular one with the size $T \times R$. The action of the $U(1)$ gauge part is given by

$$
S^{U(1)}=\frac{1}{N g^{2}} \int d^{2} \sigma \frac{1}{4} F_{\mu \nu}^{U(1)} F_{\mu \nu}^{U(1)}
$$

Let us evaluate the first factor in eq. (47) by taking the $A_{0}^{U(1)}=0$ gauge. Then, the Gauss law constraint means that $A_{\sigma}^{U(1)}$ is independent of $\sigma$. Also, considering the Wilson loop wrapping around the $\sigma$ direction, we see that $\theta(\tau) \equiv R A_{\sigma}^{U(1)}$ is an angular variable whose conjugate momentum $p$ is quantized to an integer. Employing the variable $\theta(\tau)$ and translating to the Hamiltonian form, we can compute the first factor as

$$
\begin{aligned}
\int \frac{\mathcal{D} A_{\mu}^{U(1)}}{\operatorname{Vol}(U(1))} e^{-S^{U(1)}} & =\int_{\theta(T)=\theta(0)} \mathcal{D} \theta(\tau) e^{-\frac{1}{2} \frac{1}{N g^{2} R} \int_{0}^{T} d \tau \dot{\theta}(\tau)^{2}} \\
& =\operatorname{Tr}\left(e^{-T \frac{1}{2} N g^{2} R p^{2}}\right) \\
& =\sum_{p \in \mathbf{Z}} e^{-\frac{R T}{2} N g^{2} p^{2}}
\end{aligned}
$$

\section{1 $Z_{S U(N) / \mathbf{Z}_{N}}^{D=2, \mathcal{N}=8}\left(T^{2}\right)$}

Now our remaining task is evaluating the second factor of eq. (47), which is performed by considering the dimensionally reduced version of the analysis in section 3 . Since the $Q$-exact structure as well as the $Q$-invariant one is preserved even after the dimensional reduction, the dimensionally reduced theory of a cohomological field theory becomes also a cohomological field theory. Further, the $U(1)$-symmetry in (31) remains nonanomalous in the dimensional reduction to two-dimensions in the case of the $S U(N)$ gauge group, because it contains no $U(1)$ factor. Thus, the arguments of the mass perturbation in section 3.2 can be applied also to the dimensionally reduced case. Then, the mass perturbation breaks the $\mathcal{N}=8$ supersymmetry to $\mathcal{N}=2$, and the partition function takes the form

$$
Z_{S U(N)}^{D=2, \mathcal{N}=8}\left(T^{2}\right)=\sum_{a l=N} Z_{S U(l) \otimes \mathbf{Z}_{a}}^{D=2, \mathcal{N}=2}\left(T^{2}\right)
$$

6 Since in section 3 the supersymmetry algebra (27) has no central charges, here we are to consider the $\mathcal{N}=8$ supersymmetry algebra without central charges as the result of the dimensional reduction. In fact, the central charges are written in the form of total derivatives, so they do not appear under the periodic boundary condition. In the infinite two-dimensional space, central charges exist and represent the topological charges which characterize stable solitonic modes in the theory. On the contrary, in the two-torus with the finite size, such solitonic modes do not exist stably. In this case, it can be considered that with respect to the sector of the zero total charge, dynamical degrees of freedom of the modes are contained in the theory, i.e. in the configurations which the path integration is performed over, and that they arise as metastable states when the sizes of the two-torus becomes large enough. Of course, a similar consideration is possible in the three-torus case in section 5 . 
In two-dimensions, by the argument similar as in the four-dimensional case, the $\mathbf{Z}_{a}$ factor of the gauge group yields $a^{2-1}=a$. Hence we have

$$
Z_{S U(N)}^{D=2, \mathcal{N}=8}\left(T^{2}\right)=\sum_{a l=N} a Z_{S U(l)}^{D=2, \mathcal{N}=2}\left(T^{2}\right)
$$

Here the $\mathcal{N}=2$ theory in two dimensions contains Higgs fields, whose zero momentum modes form continuous spectrum beginning with zero-energy because the field space of the Higgs fields is noncompact. This situation makes ambiguous the relation between the partition function and the Witten index, so we cannot go along the same line as in the four-dimensional case. However, owing to the fact that $\mathcal{N}=2 S U(l)$ SYM theory in two dimensions is a cohomological field theory, the partition function $Z_{S U(l)}^{D=2, \mathcal{N}=2}\left(T^{2}\right)$ can be evaluated. Let us see it from now.

After an appropriate field redefinition (see Appendix A), the classical action of the $\mathcal{N}=2$ SYM theory is written as the BRST exact form

$$
S=Q \int d^{2} \sigma \operatorname{tr}\left(\frac{1}{8 g^{2}} \eta[\phi, \bar{\phi}]-i \chi \Phi+2 g^{2} \chi H+\frac{1}{2 g^{2}} \psi_{\mu} D_{\mu} \bar{\phi}\right),
$$

where the BRST transformation is defined by

$$
\begin{array}{ll}
Q A_{\mu}=\psi_{\mu}, & Q \psi_{\mu}=-i D_{\mu} \phi, \quad Q \phi=0, \\
Q \chi=H, & Q H=[\phi, \chi], \\
Q \bar{\phi}=\eta, & Q \eta=[\phi, \bar{\phi}] .
\end{array}
$$

Note that the $Q$ is nilpotent up to the gauge transformation with the parameter $\phi$, which gives a cohomology to equivalent classes with respect to the gauge transformation. The field contents are as follows. $A_{\mu}$ is a two-dimensional gauge field, and $\psi_{\mu}, \eta, \chi$ together stand for fermions. $\phi$ and $\bar{\phi}$ are complex Higgs fields, and $H$ is a bosonic auxiliary field. Ghost number is assigned as -2 to $\bar{\phi},-1$ to $\eta$ and $\chi, 0$ to $A_{\mu}$ and $H,+1$ to $\psi_{\mu},+2$ to $\phi$. The ghost number conservation is nonanomalous for the same reason as in the case of the dimensional reduction of the $U(1)$-symmetry. The contribution of the path integration of the gauge field localizes in the configurations determined by

$$
\Phi \equiv-2 F_{12}=0
$$

The addition of a $Q$-exact term to the action does not change the theory, if it behaves well at infinity in the field space. Thus, we may discard the first term in eq. (51)

$$
Q \int d^{2} \sigma \operatorname{tr} \frac{1}{8 g^{2}} \eta[\phi, \bar{\phi}]
$$

Also, it can be seen that the partition function is independent of the coupling $g$ for the same reason. The localization (53) can be shown by integrating out $H$ and $\chi$ fields in the $g \rightarrow 0$ limit after the integrals of $\bar{\phi}$ and $\eta$. Then the partition function becomes

$$
\int \frac{\mathcal{D} A_{\mu}}{\operatorname{Vol}(S U(l))} \mathcal{D} \phi \mathcal{D} \psi_{\mu} \delta\left(D_{\mu} D_{\mu} \phi+\left\{\psi_{\mu}, \psi_{\mu}\right\}\right) \delta\left(D_{\mu} \psi_{\mu}\right) \delta\left(D_{1} \psi_{2}-D_{2} \psi_{1}\right) \delta\left(F_{12}\right),
$$


which indicates the declared localization. It should be noted that the localization is determined by the BRST fixed point $Q \chi=0$ after using the equation of motion of $H$. Eq. (55) is not in a suitable form for our purpose, so we will deform the theory judiciously as in the section 3 in ref. [28].

We consider the action with the addition of the $Q$-exact term

$$
S(t)=S^{\prime}+t Q \int d^{2} \sigma \operatorname{tr} \chi \bar{\phi}
$$

where $S^{\prime}$ stands for the action $S$ with the term (54) eliminated and $t$ is a parameter. If new BRST fixed points that flow in from infinity when $t$ turns on do not contribute, the deformed theory coincides with the original one. After integrating out $H, \eta, \chi$ and $\bar{\phi}$, we end up with the action in the large $t$ case

$$
S(t)=\frac{1}{2 g^{2} t} \int d^{2} \sigma \operatorname{tr}\left[F_{12}\left(D_{\mu} D_{\mu} \phi+\left\{\psi_{\mu}, \psi_{\mu}\right\}\right)-i D_{\mu} \psi_{\mu}\left(D_{1} \psi_{2}-D_{2} \psi_{1}\right)\right]+O\left(t^{-2}\right),
$$

which can be again written in the $Q$-exact form

$$
S(t)=Q\left[\frac{i}{2 g^{2} t} \int d^{2} \sigma \operatorname{tr} F_{12} D_{\mu} \psi_{\mu}+O\left(t^{-2}\right)\right] .
$$

Considering the case $t=-i u$ with $u$ large real positive, the $\phi$-integration yields $\delta\left(D_{\mu} D_{\mu} F_{12}\right)$. Using the normalizability of $F_{12}$, it means that the localization realizes at the solutions of $D_{\mu} F_{12}=0$, which contain extra components adding to the localization point of the original theory $F_{12}=0$. Arising of the extra components is a signal of the flow of new BRST fixed points from infinity. In fact, in the above process, the contribution of the $\chi$ and $\bar{\phi}$ integrals localizes the points

$$
\begin{aligned}
\chi & =\frac{1}{2 t} D_{\mu} \psi_{\mu}, \\
\bar{\phi} & =-\frac{1}{t} F_{12}+\frac{1}{2 t^{2}}\left(D_{\mu} D_{\mu} \phi+\left\{\psi_{\mu}, \psi_{\mu}\right\}\right),
\end{aligned}
$$

which appear first when $t$ turns on. So they are the new fixed points flowing in from infinity. Thus in general, the deformed cohomological field theory does not equivalent to the original one. However, there is a possibility that the BRST invariant operators with the following feature exist - in calculation of their correlators the extra components do not contribute. If there are such operators, the deformed theory coincides to the original one with respect to the restricted set of the operators. Indeed, we can find such operators. For the following BRST invariant operators

$$
\begin{aligned}
& \omega \equiv \int d^{2} \sigma \operatorname{tr}\left(-i \phi F_{12}+\psi_{1} \psi_{2}\right), \\
& \beta(\phi)=\operatorname{tr}(\text { polynomial of } \phi),
\end{aligned}
$$


we consider the unnormalized expectation value in the deformed theory

$$
\left\langle e^{\omega} \beta(\phi)\right\rangle^{\prime} \equiv \int \frac{\mathcal{D} A_{\mu}}{\operatorname{Vol}(S U(l))} \mathcal{D} \phi \mathcal{D} \psi_{\mu} \beta(\phi) e^{-S(-i u)+\omega} .
$$

Here due to the $e^{\omega}$ factor, we can take the limit $u=\infty$ without changing the behavior of the fields at the infinity, and thus the limit does not change the value of (60). Further integrating out $\phi$ and $\psi_{\mu}$, we end up with

$$
\left\langle e^{\omega} \beta(\phi)\right\rangle^{\prime}=\int \frac{\mathcal{D} A_{\mu}}{\operatorname{Vol}(S U(l))} \beta\left(-i \frac{\delta}{\delta F_{12}}\right) \delta\left(F_{12}\right),
$$

where the factor $\delta\left(F_{12}\right)$ (with a finite degree of the derivative $\frac{\delta}{\delta F_{12}}$ ) indicates no contributions of the extra components. Therefore, $\left\langle e^{\omega} \beta(\phi)\right\rangle^{\prime}$ coincides with the unnormalized expectation value in the original theory which we denote by $\left\langle e^{\omega} \beta(\phi)\right\rangle$.

Now we can manage to compute the partition function $Z_{S U(l)}^{D=2, \mathcal{N}=2}\left(T^{2}\right)=\langle 1\rangle$. Notice that $\omega$ has the ghost number +2 , and thus from the ghost number conservation we can show $\langle 1\rangle=\left\langle e^{\omega}\right\rangle$. This is equal to the $\beta=1$ case of eq. (61), so we find

$$
Z_{S U(l)}^{D=2, \mathcal{N}=2}\left(T^{2}\right)=\int \frac{\mathcal{D} A_{\mu}}{\operatorname{Vol}(S U(l))} \delta\left(F_{12}\right),
$$

which counts the number of the small gauge inequivalent configurations satisfying $F_{12}=0$. The number is unity, since the two-dimensional $S U(l)$ gauge theory has no nontrivial winding number. In the consequence we have

$$
Z_{S U(l)}^{D=2, \mathcal{N}=2}\left(T^{2}\right)=1 .
$$

It can be confirmed also by doing the concrete calculation, for example, employing the $A_{1}=0$ gauge fixing in eq. (62). Plugging this into eq.(50) we get the result

$$
Z_{S U(N)}^{D=2, \mathcal{N}=8}\left(T^{2}\right)=\sum_{a l=N} a .
$$

Finally by taking account into the $\mathbf{Z}_{N}$ factor as in the four dimensional case, the second factor $Z_{S U(N) / \mathbf{Z}_{N}}^{D=2, \mathcal{N}=8}\left(T^{2}\right)$ of eq. (47) is obtained as

$$
Z_{S U(N) / \mathbf{Z}_{N}}^{D=2, \mathcal{N}=8}\left(T^{2}\right)=\frac{1}{N} Z_{S U(N)}^{D=2, \mathcal{N}=8}\left(T^{2}\right)=\sum_{a \mid N} \frac{1}{a} .
$$

\subsection{Result of IIA Matrix String Partition Function}

Now we can write down the result of the partition function of the IIA matrix string theory. Substituting eqs. (48) and (65) into eq. (47), we have

$$
Z_{\text {IIA-MS }}=\left(\sum_{a \mid N} \frac{1}{a}\right) \sum_{p \in \mathbf{Z}} e^{-\frac{R T}{2} N g^{2} p^{2}},
$$


which coincides the result obtained in the strongly coupled limit $\left(g^{2} \rightarrow \infty\right)$ by Kostov and Vanhove [13]. They have conjectured that their result holds irrespectively of the strength of the coupling, and they called this property exact quasi classics. Since our calculation has been exactly performed without any approximation, it gives a proof of the exact quasi classics.

Recalling the relations (7) and (8), we rewrite the result (66) in variables in string theory

$$
Z_{\mathrm{IIA}-\mathrm{MS}}=\left(\sum_{a \mid N} \frac{1}{a}\right) \sum_{p \in \mathbf{Z}} e^{-T \frac{N L}{4 \pi} \frac{p^{2}}{\left(g_{s} l_{s}\right)^{2}}} .
$$

There are two comments in order. First, the second factor in (67) represents a certain nonperturbative effect, which corresponds to creation and annihilation of D-particle and anti-D-particle pairs. Such phenomena as creation/annihilation of D- and anti-D- objects cannot be seen in the M(atrix) theory, because in the infinite momentum frame anti-Dparticles in the $\mathrm{M}$ (atrix) theory are integrated out and do not appear. It can be seen first after compactified to the IIA matrix string theory. Second, there is no perturbative correction in the formula (67). It agrees to nonrenormalization theorems in perturbative superstring theory by Martinec [29], which tells that the 0-, 1-, 2-, and 3-point functions of

massless string vertex operators receive no perturbative corrections in flat ten-dimensional backgrounds.

\section{IIB Matrix String Partition Function}

Here, we compute the partition function of IIB matrix string theory in the ten-dimensional IIB limit, and compare the exact result of the IKKT model by Moore-Nekrasov-Shatashvili [14. We consider the three-dimensional SYM theory with periodic boundary condition as the IIB matrix string theory for the same reason as in the IIA case. With respect to the gauge group, the $U(1)$ part of the gauge group $U(N)=U(1) \times\left(S U(N) / \mathbf{Z}_{N}\right)$ for the field contents $\left(A_{\mu}, X, \theta\right)$ corresponds to the center of mass coordinates of the strings in transverse directions in the ten-dimensional IIB limit " $R_{1} R_{2} \rightarrow \infty$ with $g_{s}$ fixed," where the $U(1)$ part of the gauge field together with that of $X$ become the transverse coordinates. Thus we fix the $U(1)$ part of the gauge field as well as the Higgs fields, and consider the partition function of the three-dimensional $\mathcal{N}=8, S U(N) / \mathbf{Z}_{N}$ SYM theory as that of the IIB matrix string theory

$$
Z_{\mathrm{IIB}-\mathrm{MS}}=Z_{S U(N) / \mathbf{Z}_{N}}^{D=3, \mathcal{N}=8}\left(T^{3}\right) .
$$

The three-torus, where the SYM theory is defined on, is taken to be rectangular of the size $T \times R_{1} \times R_{2}$, with the Euclidean signature. (We performed the Wick rotation as in the IIA case.) In this case, we can also use the dimensionally reduced version of the arguments of the mass perturbation in the four dimensions, due to the following two reasons. One is that the dimensional reduction of a cohomological field theory is also a cohomological field theory. The other is that in odd dimensions the dimensionally reduced version of the $U(1)$-symmetry in eq. (31) is anomaly free. 
Going on along the same line as in the IIA case, we have

$$
\begin{aligned}
Z_{S U(N)}^{D=3, \mathcal{N}=8}\left(T^{3}\right) & =\sum_{a l=N} Z_{S U(l) \otimes \mathbf{Z}_{a}}^{D=3, \mathcal{N}=2}\left(T^{3}\right) \\
& =\sum_{a l=N} a^{2} Z_{S U(l)}^{D=3, \mathcal{N}=2}\left(T^{3}\right) .
\end{aligned}
$$

Here, there exists a Higgs field in the three-dimensional $\mathcal{N}=2$ theory. For the same reason as before, we cannot relate the partition function directly to the Witten index. However, if considering the ten-dimensional IIB limit (21) which in fact we are interested in, we can proceed further. Note that in the large volume limit physics becomes independent of the boundary condition. So we can evaluate $Z_{S U(l)}^{D=3, \mathcal{N}=2}\left(T^{3}\right)$ by adopting a twisted boundary condition instead of the periodic boundary condition.

\section{1 $\mathcal{N}=2$ partition function with a twisted boundary condition}

Here we consider the three-dimensional $\mathcal{N}=2 S U(l)$ SYM theory with the twisted boundary condition

$$
\begin{aligned}
A_{i}\left(t, \sigma_{1}, \sigma_{2}\right) & =A_{i}\left(t+T, \sigma_{1}, \sigma_{2}\right) \\
& =P A_{i}\left(t, \sigma_{1}+R_{1}, \sigma_{2}\right) P^{-1} \\
& =Q A_{i}\left(t, \sigma_{1}, \sigma_{2}+R_{2}\right) Q^{-1}
\end{aligned}
$$

where $i=1,2$, we took the $A_{0}=0$ gauge fixing, and $P$ and $Q$ are $S U(l)$ matrices satisfying $P Q=Q P e^{2 \pi i / l}$. For example, $P$ and $Q$ can be represented as

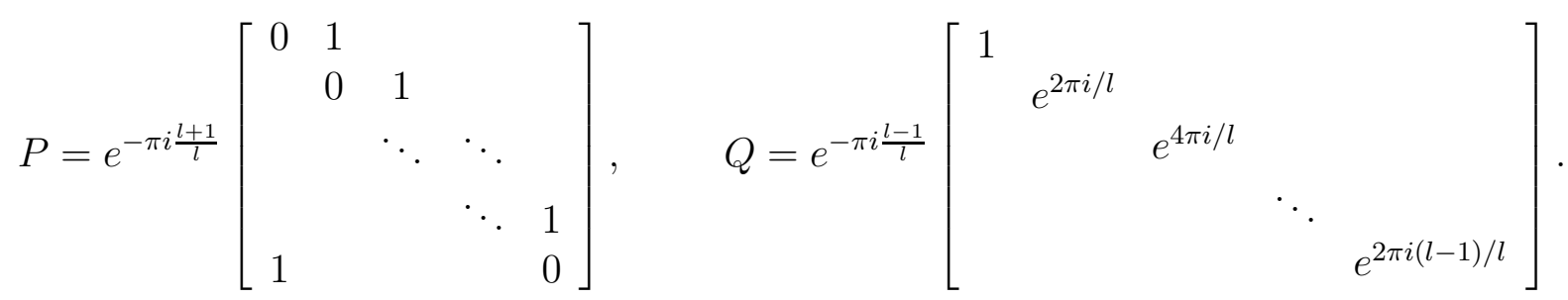

Supersymmetry requires that the other fields (Higgs $\phi$ and complex fermion $\lambda$ ) satisfy the same boundary condition as eq. (70). Under this boundary condition, zero momentum modes become trivial

$$
A_{i}^{(0)}=\phi^{(0)}=\lambda^{(0)}=0,
$$

because the constant traceless hermitian matrices commuting with $P$ and $Q$ simultaneously do not exist except the trivial case (72).

In this situation, spectra appearing in the theory are discrete, and thus the partition function coincides the Witten index. Let us consider the Witten index. The argument below is a three-dimensional analogue of Witten's consideration in the four-dimensional case [28]. Also, though it is briefly reported in [30], we will discuss it in order to make this paper more self-contained. Our problem is now reduced to counting the number of 
vacua, i.e. the number of (small) gauge inequivalent classes of the classical zero energy states. The gauge transformation preserving both of the boundary condition (70) and the gauge condition $A_{0}=0$ is generated by the time-independent $S U(l)$ matrix of the following boundary condition

$$
\begin{aligned}
U\left(\sigma_{1}, \sigma_{2}\right) & =e^{2 \pi i k_{1} / l} P U\left(\sigma_{1}+R_{1}, \sigma_{2}\right) P^{-1} \\
& =e^{2 \pi i k_{2} / l} Q U\left(\sigma_{1}, \sigma_{2}+R_{2}\right) Q^{-1}
\end{aligned}
$$

where $k_{i}=0,1, \cdots, l-1$. The classical zero energy state is given by the configuration

$$
A_{i}=-i\left(\partial_{i} U\right) U^{-1}
$$

with the other fields nil. Here, if this $U$ can be continuously deformed to the identity, there are no nontrivial sectors, and thus the vacuum is unique modulo small gauge transformations. We will see that it is in fact so. The $U$ can be written by the $S U(l)$ matrix $\tilde{U}$ satisfying the simpler boundary condition

$$
U=\left(Q^{-1}\right)^{k_{1}} P^{k_{2}} \tilde{U}
$$

where

$$
\begin{aligned}
\tilde{U}\left(\sigma_{1}, \sigma_{2}\right) & =P \tilde{U}\left(\sigma_{1}+R_{1}, \sigma_{2}\right) P^{-1} \\
& =Q \tilde{U}\left(\sigma_{1}, \sigma_{2}+R_{2}\right) Q^{-1}
\end{aligned}
$$

We consider a topological classification of gauge transformations with the boundary condition (75). Because of $\pi_{0}(S U(l))=0$, by a suitable continuous translation in a group manifold of $S U(l)$, we can always start with $\tilde{U}(0,0)=1$. Then, using eq. (75) we see that

$$
\tilde{U}(0,0)=\tilde{U}\left(R_{1}, 0\right)=\tilde{U}\left(0, R_{2}\right)=\tilde{U}\left(R_{1}, R_{2}\right)=1,
$$

i.e. $\tilde{U}$ 's on the vertices of a square with the size $R_{1} \times R_{2}$ in $\left(\sigma_{1}, \sigma_{2}\right)$-space are all identity. From the fact $\pi_{1}(S U(l))=0, \tilde{U}$ on the edges of the square can be taken identity by a continuous deformation. Further, using $\pi_{2}(S U(l))=0$, we continuously deform to $\tilde{U}=1$ everywhere in the square. Using $\pi_{0}(S U(l))=0$ again, by a continuous translation the $U$ in eq. (74) can be taken identity on the square. Thus, it is confirmed that there is no nontrivial topological vacuum sector. We conclude that the Witten index is unity, which leads to

$$
\left.Z_{S U(l)}^{D=3, \mathcal{N}=2}\left(T^{3}\right)\right|_{\text {twisted B.C. }}=1 .
$$

\subsection{Result of IIB Matrix String Partition Function}

As discussed before, in the ten-dimensional IIB limit we can replace the value of the partition function $Z_{S U(l)}^{D=3, \mathcal{N}=2}\left(T^{3}\right)$ with that of eq. (76)

$$
Z_{S U(l)}^{D=3, \mathcal{N}=2}\left(T^{3}\right)=1 .
$$


Substituting this into eq. (69), we have

$$
Z_{S U(N)}^{D=3, \mathcal{N}=8}\left(T^{3}\right)=\sum_{a l=N} a^{2},
$$

and thus the partition function of the IIB matrix string theory is obtained as

$$
\begin{aligned}
Z_{\mathrm{IIB}-\mathrm{MS}} & =Z_{S U(N) / \mathbf{Z}_{N}}^{D=3, \mathcal{N}=8}\left(T^{3}\right) \\
& =\frac{1}{N^{2}} Z_{S U(N)}^{D=3, \mathcal{N}=8}\left(T^{3}\right) \\
& =\sum_{a \mid N} \frac{1}{a^{2}}
\end{aligned}
$$

which is valid in the ten-dimensional IIB limit (21). We should remark that the result (79) coincides the result of the IKKT model [9] by Moore, Nekrasov and Shatashvili [14]. It is quite nontrivial and might be a signal of the equivalence between the two IIB matrix models arising from the different roots - one is a compactification of the BFSS M(atrix) theory, and the other is a matrix regularization of the worldsheet action of type IIB superstring in Schild gauge. Also, the result (79) has neither perturbative nor nonperturbative correction. With respect to the former it agrees again with the nonrenormalization theorems in perturbative superstring theory [29].

\section{Conclusions}

We have considered the IIA and IIB matrix string theories derived from the M(atrix) theory via toroidal compactification. We have summarized that string interactions emerge as the Yang-Mills instantons and have shown that in the IIB matrix string theory the chiral spinors are correctly reproduced.

As a preparation for computation of the matrix string partition functions, we have calculated the partition function of four-dimensional SYM theory on $T^{4}$, by mapping the theory to a cohomological field theory. Here, considering the mass perturbation (32) has made the calculation easier. We have shown the invariance of the partition function under the mass perturbation in a different fashion from the argument by Vafa and Witten [23.

We have exactly computed the partition function of the IIA matrix string theory by mapping the theory into a cohomological field theory. Our result coincides with the result obtained in the infra-red limit by Kostov and Vanhove [13], and thus gives a proof of the exact quasi classics of the theory conjectured by them. The formula for the partition function receives no perturbative correction, which is in conformity with nonrenormalization theorems in perturbative superstring theory by Martinec [29]. Also, there exist some nonperturbative corrections which come from the $U(1)$ electric flux in the SYM theory and which are interpreted as creation and annihilation of D-particle and anti-D-particle pairs. Such phenomena have been reported in high energy scattering of strings in ref. [17]. It may be interesting to deepen the meaning of our result from the line of the high energy scattering. 
Further, we have evaluated the partition function of the IIB matrix string theory in the ten-dimensional IIB limit by a similar method as in the IIA case. Our result receives neither perturbative and nonperturbative corrections, which with respect to the former agrees with the nonrenormalization theorems again. Also, our result coincides with the exact result of the partition function of the IKKT model by Moore, Nekrasov and Shatashvili [14]. Although both of the IIB matrix string theory and the IKKT model are considered to give type IIB string theory, they have arised from the distinctive origins, and the relation between them have not been clarified yet. Thus our result is quite nontrivial, and may suggest the equivalence of those two models. In the IIB matrix string theory, the S-duality is well understood from a geometry of the two-torus, but the ten-dimensional Lorentz symmetry is not manifest. On the other hand, in the IKKT model side, while there is a manifest Lorentz symmetry, we have not been able to see the S-dual structure. In this situation, it seems to be an important step to explore the equivalence and to establish the precise correspondence between them toward constructing the nonperturbative definition which manifestly realizes both of the Lorentz symmetry and the S-dual structure.

\section{Acknowledgements}

The preliminary version of this work was presented in KEK theory workshop '99. The author would like to thank the organizers and participants of the workshop, and especially N. Ishibashi, S. Iso, H. Kawai, T. Kuroki, Y. Okada, K. Okuyama and A. Tsuchiya for valuable conversations and encouragements. The research of the author is supported by the Japan Society for the Promotion of Science under the Postdoctral Research Program.

\section{Note Added}

While writing up the manuscript, I received the papers [31, 32] which discuss issues close to this work. In 31] the thermodynamic partition function of the IIA matrix string theory is calculated in the $g_{s} \rightarrow 0$ limit, and in [32] some thermodynamic properties of the IIA and IIB matrix string theories are discussed. 


\section{Appendix}

\section{A Two-Dimensional $\mathcal{N}=2 S U(l)$ Super Yang-Mills The- ory as a Cohomological Field Theory}

Here we see that the cohomological field theory (51) is identical to Euclidean $\mathcal{N}=2 S U(l)$ SYM theory in two dimensions. We perform the Wick rotation for four-dimensional $\mathcal{N}=1$ $S U(l) S Y M$ theory, and then see that its dimensional reduction to two dimensions coincides with eq. (51).

First, we start with $\mathcal{N}=1 S U(l)$ SYM theory in Minkowskian four-dimensional spacetime

$$
S_{M}=\frac{1}{g^{2}} \int d^{4} \sigma \operatorname{tr}\left(-\frac{1}{4} F_{\mu \nu} F^{\mu \nu}+\frac{i}{2} \bar{\Psi} \Gamma^{\mu} D_{\mu} \Psi\right)
$$

where $\bar{\Psi}=\Psi^{T} \Gamma^{0}$, our convention of the metric is $\eta^{\mu \nu}=\operatorname{diag}(-1,+1,+1,+1)$, and the $\Gamma$-matrices satisfy

$$
\left\{\Gamma^{\mu}, \Gamma^{\nu}\right\}=-2 \eta^{\mu \nu}
$$

After defining the $\gamma$-matrices as $\gamma_{i}=\gamma^{i}=-i \Gamma^{0} \Gamma^{i}(i=1,2,3)$ which satisfy

$$
\left\{\gamma^{i}, \gamma^{j}\right\}=-2 \delta^{i j}
$$

we perform the Wick rotation as $\sigma^{0}=-i \sigma^{4}$. Then, we have the following Euclidean action

$$
S_{E}=\frac{1}{g^{2}} \int d^{4} \sigma \operatorname{tr}\left[\frac{1}{4}\left(F_{\mu \nu}\right)^{2}+\frac{1}{2} \Psi^{T}\left(\gamma_{i} D_{i}+D_{4}\right) \Psi\right] .
$$

On the other hand, in eq. (51), integrating out $H$ and reviving the gauge fields as

$$
\phi=A_{3}+i A_{4}, \quad \bar{\phi}=A_{3}-i A_{4},
$$

it can be easily seen that the bosonic part of the action is the dimensional reduction of

$$
\int d^{4} \sigma \operatorname{tr} \frac{1}{4 g^{2}}\left(F_{\mu \nu}\right)^{2}
$$

Also, after defining the spinor in four dimensions as

$$
\Psi \equiv\left(\begin{array}{c}
\psi_{1} \\
\psi_{2} \\
\frac{1}{2} \eta \\
2 g^{2} \chi
\end{array}\right)
$$

the fermion part takes the form of the dimensional reduction of

$$
\int d^{4} \sigma \operatorname{tr} \frac{1}{2 g^{2}} \Psi^{T}\left(\gamma_{i} D_{i}+D_{4}\right) \Psi
$$


where $\gamma_{i}$ 's are

$$
\gamma_{1}=-i\left[\begin{array}{cc}
0 & \mathbf{1}_{2} \\
\mathbf{1}_{2} & 0
\end{array}\right], \quad \gamma_{2}=\left[\begin{array}{cc}
0 & -\sigma_{y} \\
\sigma_{y} & 0
\end{array}\right], \quad \gamma_{3}=i\left[\begin{array}{cc}
\mathbf{1}_{2} & 0 \\
0 & -\mathbf{1}_{2}
\end{array}\right],
$$

and satisfy eq. (81). Thus it shows that the cohomological field theory (51) coincides with the dimensional reduction of (82) to two dimensions. 


\section{References}

[1] E. Witten, String theory dynamics in various dimensions, Nucl. Phys. B443 (1995) 85, hep-th/9503124.

[2] J. Polchinski, Dirichlet-branes and Ramond-Ramond charges, Phys. Rev. Lett. 75 (1995) 4724, hep-th/9510017.

[3] E. Witten, Bound states of strings and p-branes, Nucl. Phys. B460 (1996) 335, hep-th/9510135.

[4] T. Banks, W.Fischler, S. Shenker and L. Susskind, $M$ theory as a matrix model: $a$ conjecture, Phys. Rev. D55 (1997) 5112, hep-th/9610043.

[5] W. Taylor, D-brane field theory on compact spaces, Phys. Rev. Lett. 77 (1996) 394, hep-th/9611042.

[6] R. Dijkgraaf, E. Verlinde and H. Verlinde, Matrix string theory, Nucl. Phys. B500 (1997) 43, hep-th/9703030.

[7] T. Banks and N. Seiberg, Strings from matrices, Nucl. Phys. B497 (1997) 41, hep-th/9702187.

[8] S. Sethi and L. Susskind, Rotational invariance in the M(atrix) formulation of type IIB theory, Phys. Lett. B400 (1997) 265, hep-th/9702101.

[9] N. Ishibashi, H. Kawai, Y. Kitazawa and A. Tsuchiya, A large- $N$ reduced model as superstring, Nucl. Phys. B498 (1997) 467, hep-th/9612115.

[10] T. Banks, N. Seiberg and S. Shenker, Branes from matrices, Nucl. Phys. B490 (1997) 91, hep-th/9612157.

[11] N. Seiberg, Notes on theories with 16 supercharges, hep-th/9705117.

[12] S. Paban, S. Sethi and M. Stern, Summing up instantons in three-dimensional YangMills theories, hep-th/9808119.

[13] I. Kostov and P. Vanhove, Matrix string partition functions, Phys. Lett. B444 (1998) 196, hep-th/9809130.

[14] G. Moore, N. Nekrasov and S. Shatashvili, D-particle bound states and generalised instantons, hep-th/9803265.

[15] W. Fishler, E. Halyo, A. Rajaraman and L. Susskind, The incredible schrinking torus, Nucl. Phys. B501 (1997) 409, hep-th/9703102. 
[16] T. Wynter, Gauge fields and interactions in matrix string theory, Phys. Lett. B415 (1997) 349, hep-th/9709029.

[17] S. Giddings, F. Hacquebord and H. Verlinde, High energy scattering and D-pair creation in matrix string theory, Nucl. Phys. B537 (1999) 260, hep-th/9804121.

[18] G. Bonelli, L. Bonora and F. Nesti, Matrix string theory, 2D SYM instantons and affine Toda systems, Phys. Lett. B435 (1998) 303, hep-th/9805071; G. Bonelli, L. Bonora and F. Nesti, String interactions from matrix string theory, Nucl. Phys. B538 (1999) 100; G. Bonelli, L. Bonora, F. Nesti and A. Tomasiello, Matrix string theory and its moduli space, hep-th/9901093.

[19] P. Aspinwall, Some relationships between dualities in string theory, in Proceedings of 'S-duality and mirror symmetry', Trieste 1995, Nucl. Phys. Proc. 46 (1996) 30, hep-th/9508154.

[20] J. H. Schwarz, Lectures on superstring and M theory dualities, hep-th/9607201; J. H. Schwarz, An SL(2,Z) multiplet of type IIb superstrings, Phys. Lett. 360B (1995) 13, hep-th/9508143.

[21] J. Wess and J. Bagger, Supersymmetry and supergravity, (Princeton Univ. Press, Princeton, 1982).

[22] E. Witten, Topological Quantum Field Theory, Commun. Math. Phys. 117 (1988) 353.

[23] C. Vafa and E. Witten, A strong coupling test of S-duality, Nucl. Phys. 431 (1994) 3, hep-th/9408074.

[24] J. M. F. Labastida and M. Marino, Twisted Baryon number in $\mathcal{N}=2$ supersymmetric QCD, Phys. Lett. B400 (1997) 323, hep-th/9702054.

[25] E. Witten, Supersymmetric Yang-Mills theory on a four-manifold, J. Math. Phys. 35 (1994) 5101, hep-th/9403135.

[26] J. M. F. Labastida and M. Marino, Mass perturbations in twisted $\mathcal{N}=4$ supersymmetric gauge theories, Nucl. Phys. B518 (1998) 37, hep-th/9711132.

[27] E. Witten, Constraints on supersymmetry breaking, Nucl. Phys. 202 (1982) 253.

[28] E. Witten, Two dimensional gauge theory revisited, J. Geom. Phys. 9 (1992) 303, hep-th/9204083.

[29] E. Martinec, Nonrenormalization theorems and fermionic string finiteness, Phys. Lett. B171 (1986) 189.

[30] I. Affleck, J. Harvey and E. Witten, Instantons and (super-) symmetry breaking in (2+1) dimensions, Nucl. Phys. 206 (1982) 413. 
[31] G. Grignani and G. W. Semenoff, Thermodynamic partition function of matrix superstrings, hep-th/9903246.

[32] J. P. Peñalba, Non-perturbative thermodynamics in matrix string theory, hep-th/9904094. 